\title{
CINÉTICA DO PASSO E DO TROTE DE CAVALOS TREINADOS COM E SEM RÉDEA PESSOA
}

\author{
D. A. da Silva ${ }^{1 *}$, K. Oliveira ${ }^{1}$, A. M. Pereira ${ }^{2}$, A. C. A. Duarte ${ }^{2}$
}

${ }^{1}$ UNESP - Universidade estadual Paulista, Faculdade de Ciências Agrarias e Tecnológicas, Dracena, SP, Brasil

${ }^{2}$ UFRRJ - Universidade Federal Rural do Rio de Janeiro, Seropédica, RJ, Brasil

Article history: Received 25 May 2018; Received in revised form 13 July 2018; Accepted 02 August 2018; Available online 30 September 2018.

\section{RESUMO}

O objetivo deste estudo foi avaliar o emprego da rédea Pessoa, em cavalos atletas, por meio da análise cinética do passo e do trote. Foram utilizados 12 cavalos com idade de cinco anos, pesando em média $400 \mathrm{~kg}$. Utilizou-se dois tratamentos, no primeiro grupo, os cavalos foram treinados a guia sem o uso da rédea Pessoa e no segundo tratamento realizou-se o treino mediante uso da rédea Pessoa. O delineamento experimental utilizado foi inteiramente casualizado, com seis repetições por tratamento. As variáveis monitoradas foram as forças: dorso-ventral (FDV), de propulsão (FP), médio-lateral (FML) e total (FT) nos andamentos passo e o trote. Foi verificada ausência de melhora significativa nos parâmetros cinéticos avaliados no andamento ao passo para os cavalos treinados sem a rédea Pessoa. Para os cavalos treinados com rédea Pessoa verificou-se aumentos significativos nas forças de FDV e FP, no andamento ao passo, obtendo valores médios finais de 0,84 e 1,49 W/kg, respectivamente. Observou-se melhora no desenvolvimento dos três componentes de força como FDV, FP e FT $(\mathrm{P}<0,05)$, no andamento ao trote, somente para os cavalos treinados com a rédea Pessoa. Concluiu-se que o treinamento com a rédea Pessoa modificou a cinética de deslocamento dos andamentos passo e trote em equinos. E que o trote de cavalos, submetidos ao treinamento com rédea Pessoa, apresentou maior propulsão, bem como melhoras das forças dorso-ventral e total.

Palavras-chave: equinos, propulsão, rédea auxiliar

\section{KINETICS OF TRAINED HORSES WITH PESSOA'S REIN}

\begin{abstract}
The objective of this study was to evaluate the use of the Pessoa rein, in athlete horses, through the kinetic analysis of the walk and the trot gaits. Twelve horses, aged five years and weighing an average of $400 \mathrm{~kg}$, were used. Two treatments were used, in the first group, the horses were trained the guide without the use of the Pessoa rein and in the second treatment the training was done using the Pessoa rein. The experimental design was completely randomized, with six replicates per treatment. The variables monitored were: dorso-ventral (DVF), propulsion (PF), mid-lateral (MLF) and total (TF) forces in the walk and trot gaits. There was no significant improvement in the kinetic parameters evaluated in the walk gait for

\footnotetext{
* daniellesilva.zootecnia@ outlook.com

Trabalho apresentado no $3^{\circ}$ Seminário de Construção Rural e Ambiência Aplicados à Produção Animal, 2018.
} 
horses trained without the rein Person. For horses trained with rein Person, there were significant increases in DVF and PF forces in walk gait, obtaining final mean values of 0.84 and $1.49 \mathrm{~W} / \mathrm{kg}$, respectively. There was an improvement in the development of the three components of forces, such as DVP, PF and TF ( $<<0.05)$, at trot gait, only for horses trained with the Pessoa rein. It was concluded that the training with rein Pessoa modified the kinetics of displacement of the walk and trot gaits in horses. And that the trot of horses, submitted to the rein with Person training, presented greater propulsion, as well as improvements of the dorso-ventral and total forces.

Keywords: dran reins, equines, propulsion

\section{INTRODUÇÃO}

As rédeas auxiliares têm sido empregadas às rotinas de exercícios por trabalharem no engajamento e na musculatura dorsal dos equinos, além de abreviarem o tempo necessário de treino (BAYLEY, 2010). De acordo com COTTRIALL et al. (2009), a rédea Pessoa pode incentivar o maior uso dos membros pélvicos por permitir aos equinos posição de cabeça/pescoço baixa, ou seja, mantendo a cabeça no mesmo nível ou abaixo da cernelha, e com maior ângulo de cabeça/pescoço.

A rédea Pessoa foi elaborada para o trabalho à guia de cavalos com algum nível de equitação, aplica-se a animais com boa musculatura e também àqueles que apresentam problemas no dorso, sendo utilizada com a finalidade de auxiliar na construção e manutenção da musculatura equina. A mesma constituiu-se de cordões laterais, mosquetões e um dispositivo de borracha, que serve como "tensionador", e é colocado acima do tarso, na altura da patela do animal. O tensionador encoraja o cavalo a trazer seus membros pélvicos para baixo de seu corpo e os cordões laterais abaixam a cabeça/pescoço, resultando

\section{MATERIAL E MÉTODOS}

A presente pesquisa foi certifi cada pela "Comissão de Ética em Uso de Animais", sob no 16/2011, estando de acordo com os princípios éticos de experimentação animal. Foram utilizados 12 cavalos com idade de cinco anos, pesando em média $400 \mathrm{~kg}$, sem sinais de claudicação ou de lesões músculo- respectivamente, no engajamento dos membros pélvicos e no desenvolvimento da musculatura dorsal (BAYLEY, 2010).

Segundo BROMILEY (2010), o movimento ginástico responsável por produzir impulsão, resulta da soma de duas ações biomecânicas; do engajamento provocado pelo deslocamento cranial do membro pélvico (cinemática) e da força de propulsão ocorrida durante apoio do membro pélvico no solo (cinética). Assim, a principal condição biomecânica que se procura produzir nos equinos de esporte, obtida pelo treino, é o engajamento dos membros pélvicos, que melhora a impulsão de deslocamento, sendo esta característica desejada pela Federação Equestre Internacional - FEI (HARRIS \& CLEGG, 2006; HIGGINS, 2009).

Adicionalmente, o estudo da cinética de cavalos em deslocamento, auxilia no monitoramento da qualidade dos programas de exercícios, que estão disponíveis para melhorar a performance esportiva. Neste sentido, o objetivo deste estudo foi avaliar o emprego da rédea Pessoa em cavalos atletas, por meio da análise cinética do passo e do trote.

esqueléticas e condicionados ao trabalho à guia em redondel. $\mathrm{O}$ delineamento experimental utilizado foi inteiramente casualizado com seis repetições por tratamento. Utilizou-se dois tratamentos; no primeiro grupo os cavalos foram treinados a guia sem o uso da rédea Pessoa 
e no segundo tratamento realizou-se o treino mediante uso da rédea Pessoa.

Os animais foram exercitados duas vezes por semana, por um período de três meses, em trabalho de chão à guia, no redondel, sem ou com o uso da rédea Pessoa (Figura 1). O exercício foi composto por 20 minutos de trote, dividido nos sentidos horário e anti-horário, e dez minutos a galope, realizado também nos dois sentidos, totalizando assim em trinta minutos por sessão de treino. A análise cinética foi realizada no início do experimento e após três meses de treino, mediante uso do equipamento Equimetrix, fixado ao peitoral dos animais (BARREY. et al., 2002).

Previamente as avaliações, os cavalos foram aquecidos por 15 minutos, nos andamentos ao passo e trote, guiados pelo cabresto, para depois serem conduzidos à pista de areia para mensuração (JANURA et al., 2010). Foram realizadas avaliações ao passo em velocidade média de $1,3 \mathrm{~m} / \mathrm{s}$ e no trote à 8 $\mathrm{m} / \mathrm{s}$, monitorado por fotocélula, de acordo com JANURA et al. (2010). Desta forma, cada deslocamento dos cavalos, em cada andamento, foi gravado por no mínimo 25 segundos, mediante uso de acelerômetro, equimetrix. Durante as avaliações, os cavalos foram guiados pelo cabresto ao passo e depois no trote, em linha reta, em pista plana de areia, por 25 segundos no mínimo. Assim, cada cavalo foi avaliado três vezes/andamento.

As variáveis monitoradas foram as forças dorso-ventral (FDV), de propulsão (FP), médio-lateral (FML) e total (FT) nos andamentos passo e o trote. $\mathrm{O}$ valor da diferença, entre as avaliações final e inicial, das variáveis cinéticas foram submetidas à análise de variância do programa computacional SAS (2000). Para as variáveis obtidas antes e depois no mesmo cavalo foram comparadas por meio do teste " $t$ " pareado a 0,05 de probabilidade.

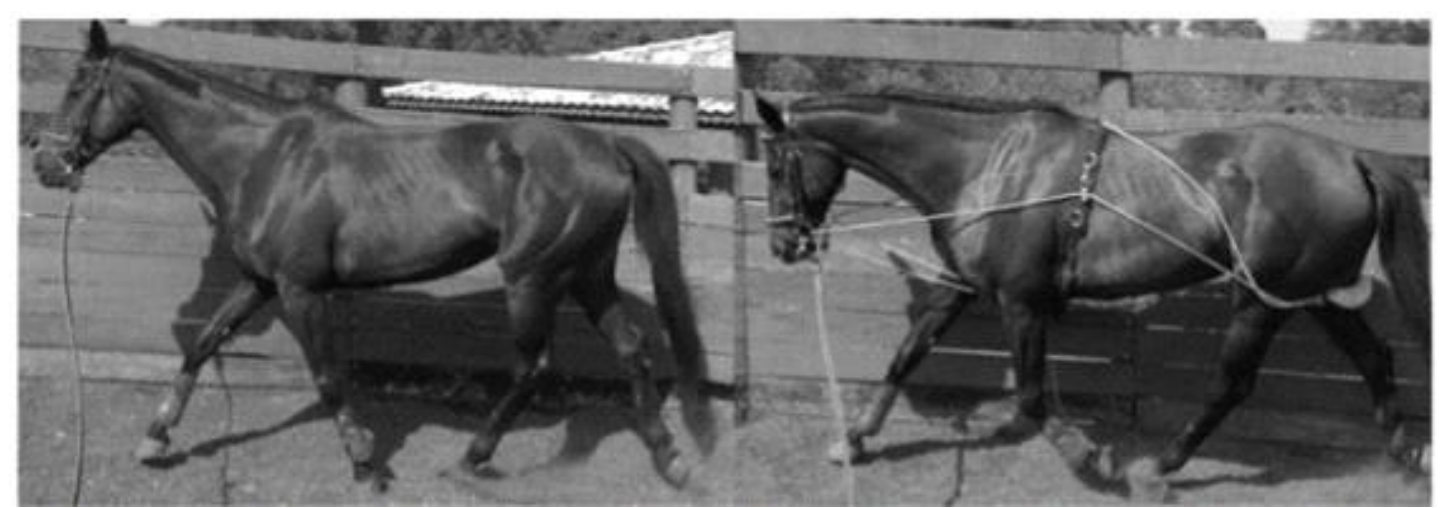

Figura 1- Ilustração do trabalho à guia em redondel, no andamento ao trote, sem rédea Pessoa (esquerda) e com rédea Pessoa (direita).

\section{RESULTADOS E DISCUSSÃO}

Na tabela 1 estão apresentados os valores médios das variáveis cinéticas no andamento ao passo, de cavalos submetidos ou não, ao treinamento com a rédea Pessoa. Após análise dos resultados foi verificada ausência de melhora significativa, aos cavalos treinados sem a rédea Pessoa, aos parâmetros cinéticos avaliados no andamento ao passo.

Tabela 1 - Valores médios da cinética de equinos no andamento ao passo, 
avaliados no início e ao final do período experimental, após treinamento sem e com rédea Pessoa.

\begin{tabular}{l|c|c|c|c}
\hline \multirow{2}{*}{$\begin{array}{c}\text { Variável } \\
(\mathrm{W} / \mathrm{kg})\end{array}$} & \multicolumn{2}{c|}{ Avaliação } & \multirow{2}{*}{ Valor de P* } & \multirow{2}{*}{ CV $(\%)$} \\
\cline { 2 - 4 } & Inicial & Final & & \\
\hline \multicolumn{5}{c}{ Sem uso da rédea Pessoa } \\
\hline Força dorso-ventral & 0,52 & 0,72 & 0,1046 & 34,46 \\
Força de propulsão & 1,07 & 1,05 & 0,9398 & 33,58 \\
Força médio-lateral & 1,48 & 1,48 & 1,0000 & 36,09 \\
Força total & 3,18 & 3,30 & 0,8791 & 38,13 \\
\hline \multicolumn{5}{c}{ Com uso da rédea Pessoa } \\
\hline Força dorso-ventral & 0,51 & 0,84 & 0,0001 & 35,27 \\
Força de propulsão & 1,09 & 1,49 & 0,0050 & 33,26 \\
Força médio-lateral & 1,54 & 1,59 & 0,7955 & 38,27 \\
Força total & 3,28 & 3,87 & 0,1068 & 30,14 \\
\hline
\end{tabular}

$\mathrm{CV}=$ coeficiente de variação

Os valores cinéticos do andamento ao passo obtidos na atual pesquisa, para cavalos treinados com rédea Pessoa, encontram-se semelhantes aos resultados encontrados na literatura consultada, para cavalos de diferentes raças (LÓPEZSANROMÁN et al., 2012). Contudo, valores superiores aos observados no presente estudo foram encontrados por BIAU et al. (2002), trabalhando com cavalos de salto e adestramento, encontraram valor médio, no andamento ao passo, para força total de $4,1 \mathrm{~W} / \mathrm{kg}$, de equinos treinados sem uso de rédea auxiliar.

Aos cavalos treinados com rédea Pessoa verificou-se aumentos significativos nas forças dorso-ventral e de propulsão, obtendo valores médios finais de 0,84 e $1,49 \mathrm{~W} / \mathrm{kg}$, respectivamente, para o andamento ao passo. A força de propulsão ao passo de cavalos treinados com rédea Pessoa, desse estudo, foram superiores aos encontrados por LÓPEZSANROMÁN et al. (2012) de 1,07 W/kg. Podendo assim dizer que os cavalos treinados com rédea Pessoa tendem a desenvolver maior força no deslocamento dorso-ventral e de propulsão, no andamento ao passo, como consequência do melhor engajamento dos membros pélvicos (WEISHAUPT et al., 2006).

Resultados superiores aos encontrados no presente estudo foram observados por BARREY et al. (2002), utilizando cavalos de adestramento, onde o valor da força dorso-ventral verificada foi de $1,13 \mathrm{~W} / \mathrm{kg}$. Esta divergência nos resultados obtidos entre as pesquisas devese as raças dos cavalos utilizados, bem como ao nível de equitação dos mesmos. Assim, os valores inferiores observados na atual pesquisa já eram esperados, pois os cavalos utilizados possuem nível básico de equitação, enquanto os animais da pesquisa supracitada pertencem a grupo de adestramento de alto rendimento esportivo.

$\mathrm{Na}$ tabela 2 estão apresentados os valores da diferença entre as medidas, final e inicial, das variáveis cinéticas do andamento ao trote, de cavalos submetidos ou não, ao treinamento com a rédea Pessoa.

Tabela 2 - Valores da diferença entre a medida final e inicial da cinética, no 
andamento ao trote, de cavalos submetidos à atividade física

\begin{tabular}{l|c|c|c|c}
\hline \multirow{2}{*}{$\begin{array}{c}\text { Variável } \\
(\mathrm{W} / \mathrm{kg})\end{array}$} & \multicolumn{2}{|c|}{ Tratamentos } & Valor de P & CV \\
\cline { 2 - 3 } & $\begin{array}{c}\text { Sem Rédea } \\
\text { Pessoa }\end{array}$ & $\begin{array}{c}\text { Com Rédea } \\
\text { Pessoa }\end{array}$ & & \\
\hline Força dorso-ventral & 1,07 & 4,27 & 0,0010 & 16,09 \\
Força de propulsão & 0,68 & 2,79 & 0,0002 & 10,53 \\
Força médio-lateral & 0,33 & 0,71 & 0,4287 & 15,96 \\
Força total & 1,50 & 6,38 & 0,0001 & 18,79 \\
\hline
\end{tabular}

$\mathrm{CV}=$ coeficiente de variação

Observou-se melhora no desenvolvimento dos três componentes de força; dorso-ventral, propulsão e total $(\mathrm{P}<0,05)$ no andamento ao trote dos cavalos treinados com a rédea Pessoa. Isto demonstra que o treinamento com a rédea Pessoa aumenta a força de deslocamento ao trote, em relação às duas forças axiais, tornando o trote mais impulsionado durante o deslocamento, o que melhora a reunião do cavalo.

Adicionalmente, o valor da força total ao trote, na avaliação final, aos cavalos treinados sem e com rédea Pessoa (presente pesquisa), obtiveram

média de 21,12 e $27,04 \quad \mathrm{~W} / \mathrm{kg}$, respectivamente. Tais resultados foram superiores aos valores encontrados com

\section{CONCLUSÕES}

$\mathrm{O}$ treinamento com a rédea Pessoa modificou a cinética de deslocamento dos andamentos passo e trote em equinos. $\mathrm{O}$ trote de cavalos, submetidos ao

\section{AGRADECIMENTOS}

A Faculdade de Ciências Agrárias e Tecnológicas (FCAT) Campus de Dracena e ao Núcleo de Extensão Ensino em

\section{REFERÊNCIAS}

BARREY, E.; DESLIENS, F.; POIREL, D.; BIAU, S. Early evaluation of dressage ability in different breeds. Equine Veterinary Journal, v. 34(Suppl.): 319324, 2002. cavalos de sela de 15,2, 21,6 e 18,0 W/kg, treinados com as rédeas back lift, chambon e elástica, respectivamente (BIAU et al, 2002).

A escassez de pesquisas publicadas na área da cinética do deslocamento de equinos $\mathrm{e}$ as diferentes metodologias utilizadas para suas mensurações limitam o aprofundamento da discussão dos resultados observados (BURLA et al., 2014). Contudo, diante dos resultados encontrados pode-se dizer que o uso da rédea Pessoa no treinamento de cavalos influencia significativamente a cinética nos andamentos passo e trote, melhorando a impulsão do deslocamento, que é uma característica altamente desejável à escala de treinos preconizada pela FEI.

treinamento com rédea Pessoa, apresentou maior propulsão, e melhora nas forças dorso-ventral e total.

Equinos (Nequi) pelo apoio e auxílio durante a pesquisa.

BAYLEY, L. Groundwork training for your horse. 3. ed. Cinccinati: David \& Charles Book, 151p, 2010.

BIAU, S ; COUVE, O.; LEMAIRE, S.; BARREY, E. The effect of reins on kinetic 
variables of locomotion. Equine Veterinary Journal, Suppl v. 34: 359362, 2002.

BROMILEY, M. Equine injury, therapy and rehabilitation. 3. ed. Oxford Blackwell. Publishing Ltda, 218p, 2010.

BURLA. J. B.; OSTERTAG. A.; WESTERATH. H. S; HILLMANN. E. Gait determination and activity measurement in horses using an Accelerometer. Computers and Electronics in Agriculture, v. 102: 127133, 2014.

COTTRIAL, S. The effects of training aids on the longissimus dorsi in the equine back. Comparative Exercise Physiology, v. (3-4): 111-114, 2008.

HARRIS, M.C.; CLEGG, L. Horse riding. 1. ed. London: Dorling Kindersley, 344p, 2006.

HIGGINS, G. How your horse moves. 1. ed. Cincinnati: David \& Charles Book. 153 p, 2009.

JANURA, M.; DVORAKOVA, T.; PEHAM, C.; SVOBODA, Z. The influence of walking speed on equine back motion in relation to hippotherapy. Veterinary Medicine Austria, v. 97:1-5, 2010.

LÓPEZ -SANROMÁN A. F.J ; R. HOLMBAK- PETERSEN A, I. SANTIAGO A; I..A. GÓMEZ DE SEGURA A, E. BARREY . Gait analysis using 3D accelerometry in horses sedated with xylazine. The Veterinary Journal, v. 193:212-216, 2012.

M. A. WEISHAUPT, T. WIESTNER, K. VON PEINEN, N. WALDERN, L. ROEPSTORFFT, R. VAN WEERENS, H. MEYERS, C. JOHNSTON. Effect of head and neck position on vertical ground reaction forces and interlimb coordination in the dressage horse ridden at walk and trot on a treadmill. Equine Veterinary Journal, v. 36 (Suppl.):387-392, 2006.
STATISTICAL ANALYSIS SYSTEM SAS. SAS user's: guide statistics. Cary: $211 \mathrm{p}, 2000$. 